\section{Multimodal biometric authentication based on the fusion of finger vein and finger geometry}

\author{
Byung Jun Kang ${ }^{a}$ and Kang Ryoung Park ${ }^{\mathrm{b}}$ \\ ${ }^{a}$ Electronics and Telecommunications Research Institute, \\ 138 Gajeongno, Yueseong-gu, Daejeon, 205-700 \\ Korea \\ ${ }^{b}$ Dongguk University, Department of Electronics \\ Engineering, Biometrics Engineering Research Center, 26 \\ Pil-dong 3-ga, Jung-gu, Seoul, 100-715 Korea \\ E-mail: parkgr@dongguk.edu
}

\begin{abstract}
We propose a new multimodal biometric recognition based on the fusion of finger vein and finger geometry. This research shows three novelties compared to previous works. First, this is the first approach to combine the finger vein and finger geometry information at the same time. Second, the proposed method includes a new finger geometry recognition based on the sequential deviation values of finger thickness extracted from a single finger. Third, we integrate finger vein and finger geometry by a score-level fusion method based on a support vector machine. Results show that recognition accuracy is significantly enhanced using the proposed method. () 2009 Society of Photo-Optical Instrumentation Engineers.
\end{abstract}

[DOI: 10.1117/1.3212651]

Subject terms: multimodal biometric recognition; finger vein; finger geometry.

Paper 090313LRR received May 5, 2009; revised manuscript received Jul. 2, 2009; accepted for publication Jul. 9, 2009; published online Sep. 2, 2009.

\section{Introduction}

Biometric identification uses an automatic method to identify an individual based on unique human features. The recognition accuracy of a unimodal biometric system can be degraded by many factors, such as illumination change, pose variation, noise in the input data, etc. ${ }^{1}$ To overcome these problems, we propose a new multimodal recognition method using score level fusion of finger-vein and finger geometry recognition. In previous research, Ferrer et al. proposed hand dorsum recognition based on fusion of a hand vein and geometry feature. ${ }^{2}$ They extracted the holistic textures of a hand vein and the shape of hand with a two-dimensional (2-D) Gabor filter. However, the recognition accuracy of this method is affected by the alignment of hand and the thickness change of the vein according to air temperature or the health condition of the user. ${ }^{3}$ To overcome this problem, we use a finger-vein recognition method based on finger-vein minutiae, such as bifurcations and ending points. The position of finger-vein minutiae is invariant to variations in vein thickness and the alignment of finger. Conventional hand geometry recognition methods extracted the geometric features of the overall hand shape using landmarks such as fingertip points and the valley points between adjacent fingers. ${ }^{4}$ Thus, the size of the cap-

0091-3286/2009/\$25.00 @ 2009 SPIE

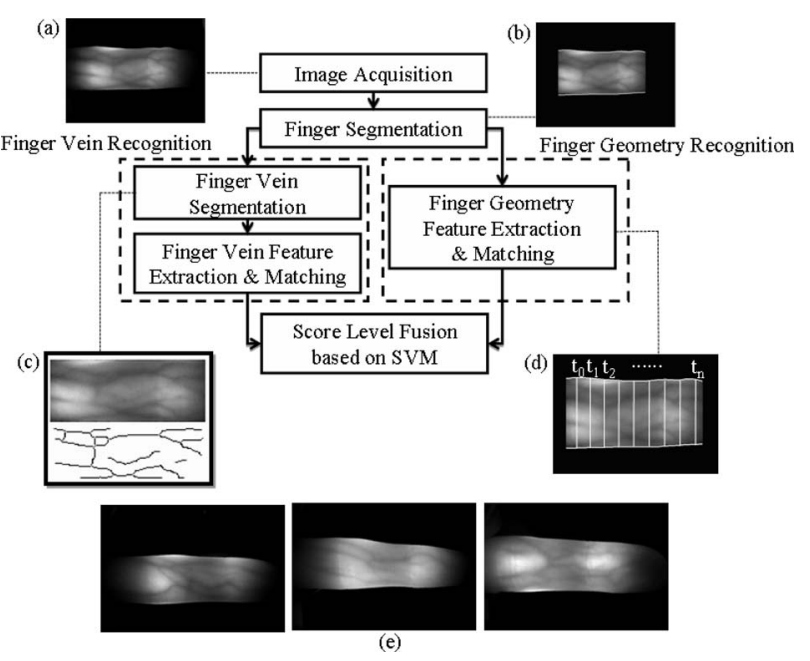

Fig. 1 Procedure of proposed multimodal biometric authentication and the examples of collected finger images.

turing device is big. However, in our research, both vein and geometric information of a finger are acquired at the same time by using a small-sized device. Thus, only part of a finger is captured in the input image and we use the change in thickness of a single finger as a geometric feature. From the information of finger vein and finger geometry, we perform score level fusion based on a support vector machine (SVM), which can greatly increase recognition accuracy.

\section{Image Acquisition, Segmentation of Finger Region, and Finger-Vein Segmentation}

Figure 1 shows the proposed multimodal biometric authentication procedure. To acquire finger-vein patterns and finger geometry information at the same time, our capturing device consists of near-infrared (NIR) light illuminators and a camera that includes an NIR light-pass filter. The NIR light illuminates the user's finger on the back side of the finger, and the image is captured by the camera located on the opposite side of the finger. ${ }^{5}$ Examples of images acquired by the device are shown in Fig. 1(a). The finger region is brighter than the background region due to NIR light that illuminates through the finger, as shown in Fig. 1(b). Therefore, the edge line between the finger and the background region is distinctive on the input image. On the basis of this result, the finger region is segmented by cal-

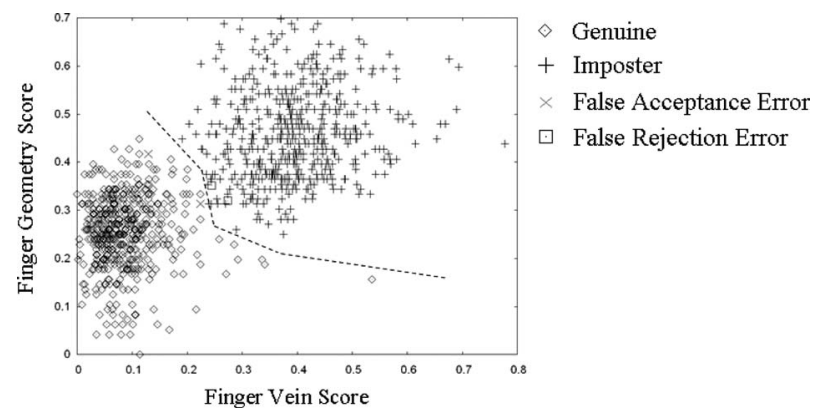

Fig. 2 SVM classification based on finger-vein score and finger geometry score. 


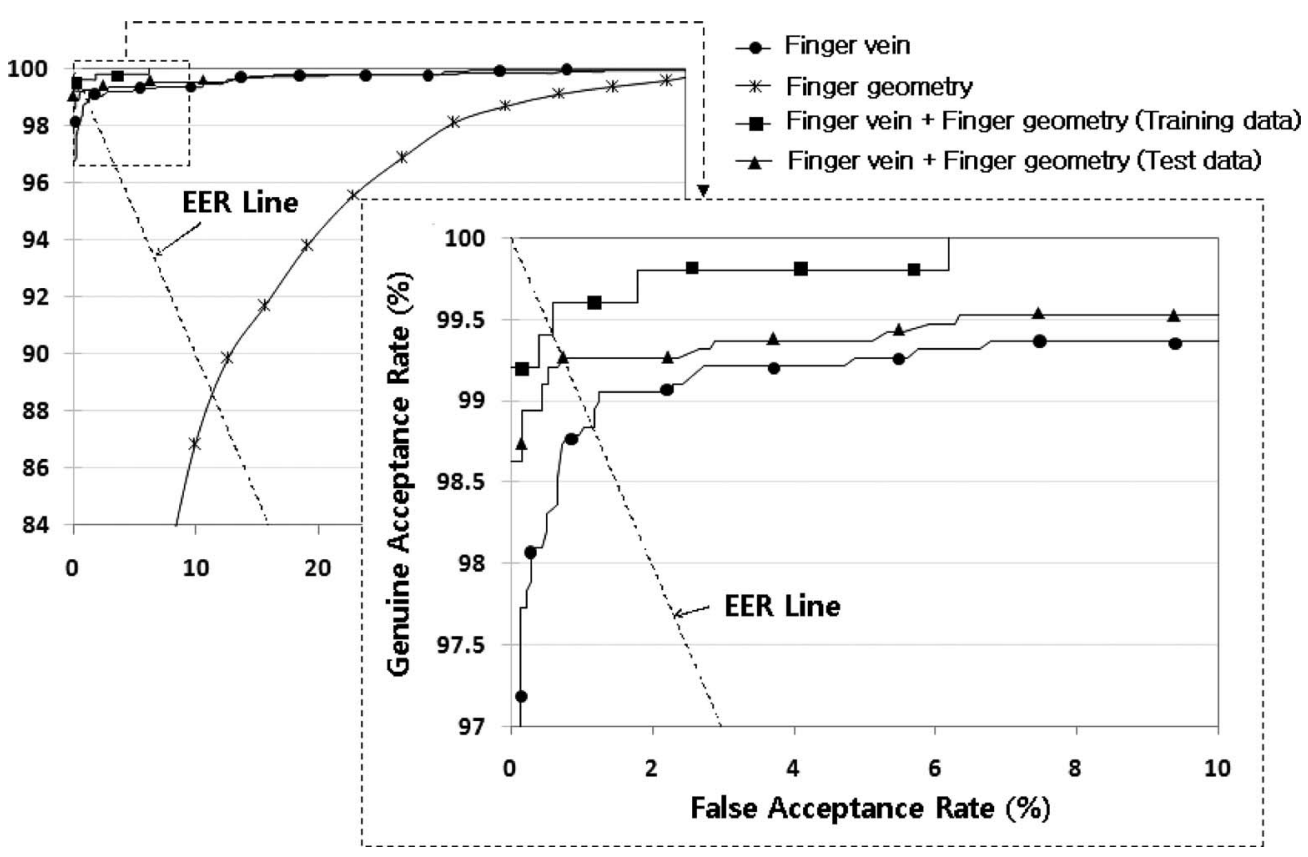

Fig. 3 ROC curves (1).

culating the gradient value in the $Y$ (vertical) direction. The segmented region of a finger vein is size normalized by stretching, as shown in Fig. 1(c). Then, the finger-vein region is binarized by adaptive local thresholding, which is described in Ref. 6. Morphological opening and closing is used to remove segmentation noise. The skeleton image is obtained by a thinning algorithm ${ }^{7}$ shown in Fig. 1(c).

\section{Feature Extraction of Finger Vein and Matching}

Minutiae points, such as the ending and bifurcation points of the finger vein, are extracted as feature points from the skeletonized vein image. They are detected by counting black pixels of eight-directional neighborhoods on each position of the skeletonized finger-vein line. On the basis of the ending and bifurcation points, the dissimilarity between the input vein feature vector and the enrolled feature vector is calculated using the modified Hausdorff distance (MHD) as in Ref. 8. Equation (1) shows the definition for a MHD. $d(X, Y)$ is the MHD between the two point sets. In the set $X=\left\{x_{1}, x_{2}, \ldots, x_{n}\right\}, x_{i}$ is one minutiae point (the ending or bifurcation point) obtained from the input image. In the set $Y=\left\{y_{1}, y_{2}, \ldots, y_{m}\right\}, y_{i}$ is one minutiae point obtained from the enrolled image. A smaller value of MHD represents that the two point sets are more similar,

$d(X, Y)=\frac{1}{n_{x_{i} \in X}} \sum_{y_{i} \in Y} \min _{y_{i}}\left\|x_{i}-y_{i}\right\|$.

\section{Feature Extraction of Finger Geometry and Matching}

The geometric feature vector of the finger consists of the sequential deviation values of finger thickness ( $T$ $\left.=\left\{\Delta t_{1}, \Delta t_{2}, \ldots, \Delta t_{n}\right\}, \Delta t_{k}=t_{k-1}-t_{k}\right)$. Here, $t_{k}$ are the vertical thicknesses of the finger region, as shown in Fig. 1(d). Because the input finger region is first aligned with the en- rolled one based on the extracted bifurcation and ending points of the finger vein, the geometric features of finger shape are not affected by the rotation and translation of the finger. The dissimilarity between input and enrolled feature vectors $\left(T_{R}\right.$ and $\left.T_{E}\right)$ is calculated by the Euclidean distance.

\section{Score-Level Fusion Based on SVM}

We discriminate between a genuine class and an imposter class using $\mathrm{SVM}^{9}$ based on a finger-vein score calculated using Eq. (1) and a finger geometry score. These two scores are normalized in the range of $0-1$ by $z$-score normalization using the mean and standard deviation of each score distribution. $^{10}$

\section{Experimental Results}

To test the proposed algorithm, we collected a database consisting of 8160 finger images from 102 people. Half the data were used for SVM training, and the other half was used for testing. Figure 1(e) shows the examples of collected finger images. We used winSVM, which found the

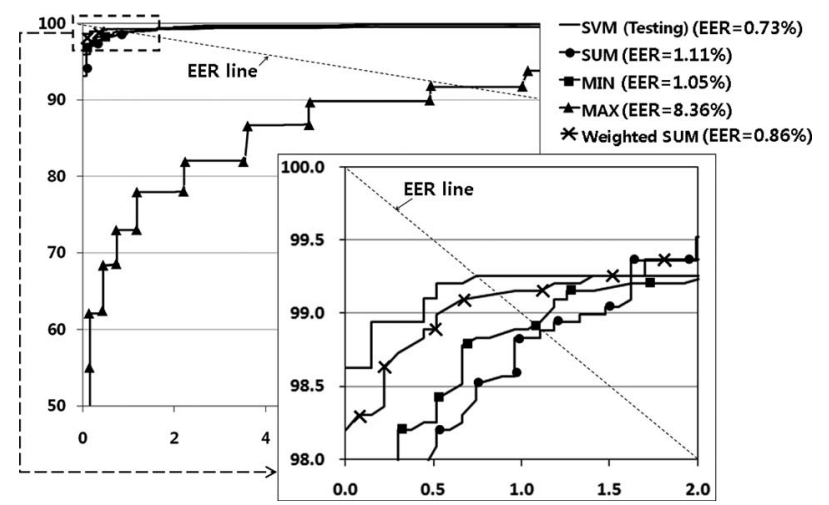

Fig. 4 ROC curves (2). 
Table 1 The changes of modified Hausdorff distance and Euclidean distance of same user's data according to the shifting and rotation of finger.

\begin{tabular}{|c|c|c|c|c|c|c|c|c|c|}
\hline & & \multicolumn{4}{|c|}{ Shift (pixels) } & \multicolumn{4}{|c|}{ Rotation (deg) } \\
\hline & & 0 & 20 & 40 & 60 & 0 & 10 & 20 & 30 \\
\hline \multirow{2}{*}{$\begin{array}{c}\text { Finger } \\
\text { vein } \\
\text { (modified } \\
\text { Hausdorff } \\
\text { distance) }\end{array}$} & $\begin{array}{l}\text { Without } \\
\text { alignment }\end{array}$ & 0.181 & 0.276 & 0.424 & 0.561 & 0.169 & 0.291 & 0.449 & 0.524 \\
\hline & $\begin{array}{c}\text { With } \\
\text { alignment }\end{array}$ & 0.18 & 0.181 & 0.181 & 0.182 & 0.168 & 0.169 & 0.169 & 0.169 \\
\hline \multirow{2}{*}{$\begin{array}{c}\text { Finger } \\
\text { geometry } \\
\text { (euclidean } \\
\text { distance) }\end{array}$} & $\begin{array}{l}\text { Without } \\
\text { alignment }\end{array}$ & 0.163 & 0.294 & 0.523 & 0.654 & 0.145 & 0.284 & 0.393 & 0.443 \\
\hline & $\begin{array}{c}\text { With } \\
\text { alignment }\end{array}$ & 0.162 & 0.164 & 0.164 & 0.165 & 0.144 & 0.144 & 0.145 & 0.145 \\
\hline
\end{tabular}

optimal parameters and kernel for SVM based on the highest classification accuracy. ${ }^{11,12}$ Experimental results showed that the highest classification accuracy of SVM was obtained with the ANOVA kernel $(\gamma=0.8, d=4)$ among various kinds of SVM kernels including dot, radial base, polynomial, neural, and ANOVA kernels. The classification results of SVM are shown in Fig. 2. From the output values of SVM, the optimal threshold was determined based on a Bayesian rule with which the minimum equal error rate (EER) of authentication was obtained. The EERs of fingervein recognition, finger geometry, and the proposed multimodal authentication (with test data) were 1.1, 12.21, and $0.73 \%$, respectively. Figure 3 shows the receiver operational characteristic (ROC) curves, from which we can know that the proposed multimodal authentication shows higher recognition accuracy than other approaches. Figure 4 shows the ROC curves, which represent the comparisons to other score-level fusion methods, such as MIN, MAX, SUM, Weighted SUM, and the proposed SVM method. We can know that the proposed multimodal authentication shows higher recognition accuracy than other approaches. In next experiment, we tested the changes of modified Hausdorff distance and Euclidean distance of the same user's data based on finger-vein and geometry features according to the shifting and rotation of the finger. As shown in Table 1, the distances were almost the same with the alignment, which showed that the proposed method was not affected by the translation and rotation of the finger.

\section{Conclusion}

In this study, we propose a new multimodal authentication approach based on a score-level fusion of finger vein and finger geometry by SVM. In future research, we plan to perform more field tests with various kinds of data acquired in an outdoor environment.

\section{Acknowledgment}

This work was supported by the Korea Science and Engineering Foundation (KOSEF) through the Biometrics Engineering Research Center (BERC) at Yonsei University [R112002105000000(2009)].

\section{References}

1. A. K. Jain, A. Ross, and S. Prabhakar, "An Introduction to biometric recognition," IEEE Trans. Circuits Syst. Video Technol. 14(1), 4-19 (2004)

2. M. A. Ferrer, A. Morales, and L. Ortega, "Infrared hand dorsum images for identification," Electron. Lett. 45(6), 306-308 (2009).

3. N. Miura, A. Nagasaka, and T. Miyatake, "Extraction of finger-vein patterns using maximum curvature points in image profiles," IEICE Trans. Inf. Syst. E90-D(8), 1185-1194 (2007).

4. A. K. Jain, A. Ross, and S. Prabhakar, "A prototype hand geometrybased verification system," Proc. Int. Conf. on Audio- and Videobased Biometric Person Authentication (AVBPA), 166-171, Springer, Washington, (1999).

5. Y. K. Jang, B. J. Kang, and K. R. Park, "A study on touchless finger vein recognition robust to the alignment and rotation of finger," KIPS Trans: Part B 15-B(4), 275-284 (2008).

6. S. Kim, K. R. Park, D. Park, and C. S. Won, "Fast detection of finger-vein region for finger-vein recognition," J. IEEK, 46(1), 23-31 (2009)

7. M. Sonka, V. Hlavac, and R. Boyle, Image Processing, Analysis, and Machine Vision, 2nd Ed., Brooks and Cole Publishing, Florence (1998)

8. L. Wang, G. Leedham, and D. S. Cho, "Minutiae feature analysis for infrared hand vein pattern biometrics," Pattern Recogn. 41, 920-929 (2008).

9. V. Vapnik, Statistical Learning Theory, Wiley, Hoboken, NJ (1998).

10. A. K. Jain, K. Nandakumar, and A. Ross, "Score normalization in multimodal biometric systems," Pattern Recogn. 38, 2270-2285 (2005).

11. B. Scholkopf, R. C. Williamson, A. J. Smola, and J. Shawe-Taylor, Neural Information Systems, Vol. 12, MIT Press, Cambridge, MA (2000).

12. winSVM, 〈http://www.cs.ucl.ac.uk/staff/M.Sewell/winsvm/〉15 June (2009). 